\title{
Effective scheduling model of data in financial management software running process
}

\author{
Sun jianchun
}

\author{
Changchun Normal University, Jilin Changchun 130032, China
}

\begin{abstract}
Keywords: financial management; data; scheduling model;
\end{abstract}
\begin{abstract}
When the financial management software is running, the data needs to be efficient scheduled. In the scheduling process, because there is a strong randomness for data utilization, traditional scheduling method is likely to cause a data collision, resulting in reduced efficiency of scheduling. This paper proposes efficient data scheduling method in financial management software running based on the collision avoidance. According to the target data transmission case, to calculate the amount of data scheduling tasks, binary search is applied on predicted result. Experimental results show that the proposed algorithm adopted for data in the scheduling financial management software running process, can effectively improve the efficiency of scheduling.
\end{abstract}

\section{Introduction}

With the rapid development of information technology, data management has begun to receive attention. Data management technology applied in the process of financial management can effectively improve the efficiency of financial management to meet the needs of financial management [1]. Thus, how to schedule the data in financial management software running process efficiently, has become a hot topic need more research in data management area, and being focused on by a lot of experts [2]. At current stage, many research institutions have research data scheduling in financial management software in-depth, and a lot of scheduling methods are proposed, including data scheduling method based on ant colony algorithm in financial management software running [3], data scheduling method based on simulated annealing algorithm in financial management software running [4] and data scheduling method based on data correlation coefficient in financial management software running [5]. Among them, the most commonly used is the scheduling method based on data correlation coefficient [6]. Due to data scheduling method in financial management software running plays an irreplaceable role in the field of data management, therefore, has broad prospects for development, regarded the key issues by many experts.

\section{Principle of data scheduling optimization methods in financial management software}

\subsection{Forecast for the quantity of data scheduling tasks in Financial Management Software}

When the data is scheduled in the financial management software running process, the time slot of data scheduling is represented by $f=[f(0), f(1), \cdots, f(Q-1)]^{T}$, the number of scheduling data is $r$. Therefore, the time slot of each data scheduling tasks need to obey the distribution $d \sim p(f, r)$, according to the distribution to predict the task quantity of data scheduling in financial management software running process accurately.

Based on the following formula to calculate the number of all the data scheduling tasks in financial management software running process:

$$
\hat{r}=\operatorname{arc} \min _{\bar{q} \in \varepsilon} \sum_{r=1}^{+\infty} M(\bar{r}, r) p(r \mid f)
$$

In the formula, the average value of $r$ is calculated, the results obtained can be expressed by $\bar{r} . \sum_{r=1}^{r} \bar{r}(r \mid f)=1$ is set, the forecast cost function of data schedule in financial management software running as follows:

$$
M(\bar{r}, r)=|\bar{r}-r|
$$

The above formula is input into the calculation of data scheduled tasks amounts in financial management software running, it is possible to obtain the following results: 
$\hat{r}=\operatorname{arc} \min _{\bar{r}}\left(\sum_{r=1}^{\bar{r}} q(r \mid r)-\sum_{r=\bar{r}}^{r} q(r \mid f)\right)$

According to the following formula can calculate maxima of predicted data running scheduled tasks amount in the k-th financial management software:

$$
M(\bar{r}, r)=\left\{\begin{array}{l}
1|\bar{r}-r|>\frac{\Delta}{2} \\
0|\bar{r}-r| \leq \frac{\Delta}{2}
\end{array}\right.
$$

Wherein, $\Delta$ is data scheduling operation status parameter in the financial management software processes, the following formula can be adopted to predict the amount of data scheduled task:

$$
r=\operatorname{arc} \max _{\bar{r}} r(\bar{r} \mid f)
$$

According to the method outlined above, data scheduled tasks amount in the process of financial management software running is eligible to be predicted, and thus provide the basis for data scheduling conflict avoidance operations.

2.2 Binary search method for data scheduled task

Data scheduled time in financial management software running can be represented by $y_{T}$, the sub-function number of software required applications in this process can be indicated by $y_{V}$, the following equation is employed to describe the above relationship:

$$
\begin{aligned}
& y=y_{T}+y_{V} \\
& y_{T}=s_{p}\left(R_{T V}+R_{T t} H\right) \\
& y_{V}=s_{p} R_{V t}+w p R_{V V}
\end{aligned}
$$

The tasks number of all data required scheduling in financial management software can be expressed by $p$, the number of time slots can be represented by $H$, the number of binary levels denoted as $N$, the following formula is utilized to calculate the number of data scheduling time slots in financial management software operation:

$H=2^{N}$

Data conflict probability can be calculated using the following formula in the process of data scheduling:

$$
\begin{aligned}
& R(E)=1-R(U)-R(K)=1-R(U)-R(K) \\
& =1-\left(1-D^{N}\right)-\sum_{m=1} R(m, p, N) R_{Q E}(H, m)
\end{aligned}
$$

Wherein, $R_{Q E}=H / H(H-m)$. During the process of searching with binary tree, the following formula is able to calculate the probability of data scheduling conflict in financial management software running process:

$$
\begin{aligned}
& Z_{y, N, p}=Z_{N, p}=1-\left(1-D^{N}\right)- \\
& \sum_{m=1} R(m, p, N) R_{Q E}(H, m)(N>0)
\end{aligned}
$$

When the number of binary tree levels is $N=0, z_{y, p}=1-R_{Q E}(H, p)$. With the following formula to calculate the probability of data scheduling conflicts appears in $y$-th financial management software sub-function of $N$ level binary tree:

$$
X_{y, N, p}=\left\{\begin{array}{c}
1(N=0) \\
Z_{N-1, p}(N>0)
\end{array}\right.
$$

According to the above description, the collision probability of the data scheduling can be calculated during the operation of the financial management software, assuming that a conflict occurs, the other data is selected for scheduling, so as to achieve the data anti-collision operation in 
financial management software running, thereby reducing the probability of conflict.

\section{Results Analysis}

In order to verify the effectiveness of the proposed method, a single experiment need to be conducted. Construction of the experimental environment with Matlab 7.1. During the experiment, the data scheduling simulation environment need to be established in financial management software running process. Data storage area distribution in data scheduled tasks can be described by the following figure:

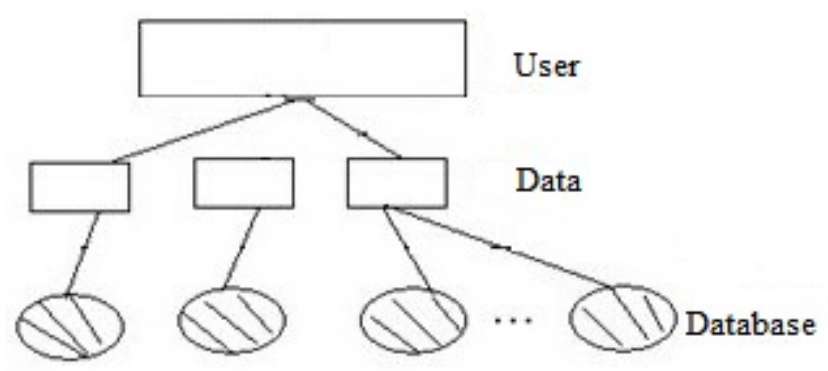

Figure 1 Distribution of the data storage area

During the experiment, the amount of data in financial management software is set to increase. In this case, the amount of data scheduling tasks is more, the probability of data scheduling conflicts is higher. With traditional algorithm and proposed algorithm to conduct data scheduling experiments in financial management software running process respectively. During the above experiment, the resource utilization can be described by the following table:

Table 1 data table of resource utilization with different algorithms

\begin{tabular}{|c|c|c|c|}
\hline \multirow{2}{*}{$\begin{array}{l}\text { Number of } \\
\text { experiments } \\
\text { (times) }\end{array}$} & \multirow[b]{2}{*}{ target data size } & \multicolumn{2}{|c|}{ Resource utilization rate (\%) } \\
\hline & & $\begin{array}{c}\text { Traditional } \\
\text { algorithm }\end{array}$ & $\begin{array}{l}\text { Improved } \\
\text { algorithm }\end{array}$ \\
\hline 1 & 1000 & 45 & 65 \\
\hline 2 & 2000 & 44 & 61 \\
\hline 3 & 3000 & 43 & 66 \\
\hline 4 & 4000 & 44 & 62 \\
\hline 5 & 5000 & 46 & 63 \\
\hline 6 & 6000 & 42 & 66 \\
\hline 7 & 7000 & 44 & 67 \\
\hline 8 & 8000 & 42 & 61 \\
\hline 9 & 9000 & 41 & 62 \\
\hline 10 & 10000 & 45 & 66 \\
\hline
\end{tabular}

According to the experimental results, it can be known that as the amount of data scheduled tasks increases in financial management software running process, with the proposed algorithm for scheduling have much higher resource utilization than traditional scheduling algorithm.

\section{Conclusion}

In the scheduling process, because there is a strong randomness for data utilization, traditional scheduling method is likely to cause a data collision, resulting in reduced efficiency of scheduling. This paper proposes efficient data scheduling method in financial management software running based on the collision avoidance. According to the target data transmission case, to calculate the amount of data scheduling tasks, binary search is applied on predicted result. Experimental results show that the proposed algorithm adopted for data in the scheduling financial management software running process, can effectively improve the efficiency of scheduling, avoided conflict situations, and achieved satisfactory results.

\section{Acknowledgement}

"Twelfth five-year" jilin province department of education and social science research (item number, educational, scientific and cultural word [2013] no. 214) of the phased achievements 


\section{References}

[1] Zhang Shudong, Cao Yuanda, Liao Lejian. Job scheduling Algorithm Based on Credit Model in Cluster Environment [J]. Mini-micro systems, 2005, 26(12):2140-2143.

[2] Wang Tianqing, Xie Jun, Zeng Zhou. Strategy research on gridding task schedule based on ant colony algorithm [J]. Computer Engineering and Design, 2007, 28(15):3611-3612.

[3] Li Ning, Chen Bin, Xu Kai. An improved grid spatial index algorithm based on navigation road network [J]. Journal of Shanghai Normal University (Natural Sciences), 2008, 37(5):482-483.

[4] Su K. F ,Ou C. H, Jiau H. C. Localization with Mobile Anchor Point s in Wireless Sensor Networks[J]. IEEE Transactions on Vehicular Technology, 2005.54: 1187-1197.

[5] P. Xie, Z. Zhou, Z. Peng, H Yan, T.S. Hu, J.-H. Cui, Z.J. Shi, Y.S. Fei and S.L. Zhou, Aqua-Sim: A NS-2 Based Simulator for Underwater Sensor Networks[C], IEEE/MTS OCEANS, 2009:1-7.

[6] Zhong Qiuxi, Chen Huowang. Genetic Operators in Task Matching and Scheduling [J]. Journal of national university of defense technology, 2000,22(3):34-38. 